\title{
Rheological Behaviours and Constitutive Models for 9Cr18Mo Stainless Steel at High Temperature and High Strain Rate
}

\author{
Haishen Jia \\ Lanzhou Institute of Technology \\ Jilin Zhang \\ Lanzhou Institute of Technology \\ Xiangbin Yi (D 530064133@qq.com ) \\ Lanzhou Institute of Technology \\ Jiancheng Shen \\ Lanzhou Institute of Technology \\ Linhu Tang \\ Lanzhou Institute of Technology \\ Chuangwen Xu \\ Lanzhou Institute of Technology \\ Wencui Luo \\ Lanzhou Institute of Technology
}

\section{Research Article}

Keywords: Rheological behavior, constitutive model, correlation coefficient, average relative error

Posted Date: June 21st, 2021

DOI: https://doi.org/10.21203/rs.3.rs-620994/v1

License: (9) This work is licensed under a Creative Commons Attribution 4.0 International License.

Read Full License 


\section{Rheological behaviours and constitutive models for 9Cr18Mo stainless steel at high temperature}

and high strain rate

Haishen Jia ${ }^{2}$, Jilin Zhang ${ }^{1}$, Xiangbin $\mathrm{Yi}^{3}$, Jiancheng Shen ${ }^{2}$, Linhu Tang ${ }^{3}$, Chuangwen Xu ${ }^{3}$, Wencui Luo ${ }^{1}$

1 Gansu Province Mechanical Equipment Advanced Manufacturing Collaborative Innovation Center, Lanzhou Institute of Technology, Lanzhou 730050, China

2 Gansu Province Precision Machining Technology and Equipment Engineering Research Center, Lanzhou Institute of Technology, Lanzhou 730050, China

3 Key Laboratory of Green Cutting Technology and Application in Gansu Province, Lanzhou Institute of Technology, Lanzhou 730050, China

\section{Corresponding author: Xiang-bin Yi_ E-mail:530064133@qq.com}

Abstract: The compression test was conducted on 9Cr18Mo stainless steel by using the UTM5305 universal testing machine and the split Hopkinson pressure bar (SHPB) test device. In this way, the stress-strain curves pertaining to quasi-static (strain rate of $0.001 \sim 0.1 \mathrm{~s}^{-1}$ ) and dynamic (temperature range of $25 \sim 650{ }^{\circ} \mathrm{C}$ and strain rate of $800 \sim 4,000 \mathrm{~s}^{-1}$ ) states were attained. According to the stress-strain curves, the rheological behaviours of $9 \mathrm{Cr} 18 \mathrm{Mo}$ stainless steel at high temperature and high strain rate were discussed. Based on the test data, the parameters of two constitutive models (Johnson-Cook (J-C) and Power-Law (PL)) for 9Cr18Mo stainless steel were identified and the correlation coefficients $(R)$ and average absolute relative errors (AAREs) of the two constitutive models were compared. The results showed that $9 \mathrm{Cr} 18 \mathrm{Mo}$ stainless steel presents strain-rate sensitivity and significant thermal softening, that is, the flow stress on $9 \mathrm{Cr} 18 \mathrm{Mo}$ stainless steel increases with strain rate while significantly reduces with increasing temperature. The $R$ values are 0.9697 and 0.9896 and the AAREs of two constitutive models are $2.77 \%$ and $1.85 \%$, respectively. Hence, the P-L constitutive model shows a higher prediction accuracy compared with the J-C constitutive model and can better describe the rheological behaviours of 9Cr18Mo stainless steel at high temperature and high strain rate.

Keywords: Rheological behavior, constitutive model, correlation coefficient, average relative error.

\section{Introduction}

Due to its high hardness, excellent corrosion resistance, and favourable wear resistance, 9Cr18Mo stainless steel is widely used in such as aerospace, ocean engineering, and the nuclear industry, especially in the manufacture of bearing parts working in a highly oxidizing atmosphere without lubrication and in corrosive environments ${ }^{[1-3]} \cdot 9 \mathrm{Cr} 18 \mathrm{Mo}$ stainless steel is quite sensitive to the choice of cutting technique as specimens are elasto-plastically deformed, torn and fractured in a thermo-mechanical coupled stress field at a high temperature and strain rate during cutting, showing various characteristics including high deformation resistance, high cutting temperature, and significant tool wear ${ }^{[4-6]}$. Thus, it is necessary to establish a constitutive model describing the rheological behaviours of $9 \mathrm{Cr} 18 \mathrm{Mo}$ stainless steel at high temperature and high strain rate, which helps when optimising the cutting parameters and guaranteeing accuracy of simulation during cutting. 
As a constitutive equation for describing change relationships of flow stress on materials with strain, strain rate and temperature, a constitutive model reveals the rheological behaviours of materials during elasto-plastic deformation. To describe the elastic-plastic deformation mechanism of materials in the cutting process, scholars have conducted numerous studies of constitutive models for materials. Typical constitutive models include Johnson-Cook (J-C), power-law (P-L), and ZerilliArmstrong (Z-A) models ${ }^{[7-9]}$. However, the applicability of the constitutive models varies for different materials. Z. He $\quad{ }^{[10]}$ et al. explored the dynamic mechanical properties of $0 \mathrm{Cr} 17 \mathrm{Ni} 4 \mathrm{Cu} 4 \mathrm{Nb}$ stainless steel by applying the Cowper-Symonds $(\mathrm{C}-\mathrm{S})$ constitutive model. The result indicated that the model can predict the stress-strain curve from the perspective of safety of engineering application. H.Z. Yan ${ }^{[11]}$ et al. performed quasi-static compression mechanical tests and orthogonal cutting tests to establish the J-C constitutive model for 20CrMo materials. By comparing the simulation result with test result, the established model has been proved to be accurate. D. Forni ${ }^{[12]}$ et al. investigated the dynamic constitutive relationship of S355 structural steel by using J-C and C-S constitutive models and assessed the prediction accuracy of the two models. B. J. Tuazon ${ }^{[13]}$ et al. modified the term for strain-rate strengthening effect of the $\mathrm{J}-\mathrm{C}$ constitutive model and fitted the constitutive equations for three high strength steels (HSA800, Hi-Mn and AISI 4340). The result indicated that the fitting effect of the modified J-C constitutive model is superior to that of the original $\mathrm{J}-\mathrm{C}$ constitutive model, especially in zones with a high strain rate. Yong Huang [14] determined the J-C constitutive model for AISI 52100 bearing steel by conducting the orthogonal cutting tests. By comparing predicted and test results, it was found that the established constitutive model was accurate. With consideration of effects of temperature and strain as well as their coupling effect on the flow stress, D. Samantaray ${ }^{[15]}$ et al. constructed the modified Z-A constitutive model, which can predict the flow stress within a large range of temperatures and strain rates.

Due to various properties including excellent wear resistance, corrosion resistance, and high hardness, $9 \mathrm{Cr} 18 \mathrm{Mo}$ stainless steel is extensively used; however, scholars have rarely investigated rheological behaviours of $9 \mathrm{Cr} 18 \mathrm{Mo}$ stainless steel at high temperature and high strain rate and the constitutive model for describing its dynamic mechanical properties. As a result, we lack an accurate description of the elasto-plastic deformation behaviours of 9Cr18Mo stainless steel to ensure accurate simulations of the cutting process. Therefore, by employing the UTM5305 universal testing machine and the split Hopkinson pressure bar (SHPB) test device, quasi-static and dynamic compression tests were performed on 9Cr18Mo stainless steel; according to the test data, the influences of temperature and strain rate on the rheological behaviours of 9Cr18Mo stainless steel were ascertained. In addition, J-C and P-L constitutive models at high temperature and high strain rate were established. Moreover, the accuracy of the established models was judged from the perspectives of the correlation coefficient $(R)$ and average absolute relative error (AARE) thereof; the research results provide certain theoretical reference and related data for analysing the cutting performance of $9 \mathrm{Cr} 18 \mathrm{Mo}$ stainless steel, optimising various technological parameters, and perfecting the finite element simulation of the cutting process.

\section{Tests}

The $9 \mathrm{Cr} 18 \mathrm{Mo}$ stainless steel used for the test was in the form of steel bars with a diameter of $30 \mathrm{~mm}$ prepared by Shanghai 
Baosteel Group Corporation, China (the chemical compositions of which is provided in Table 1). Before the test, the materials were prepared into cylindrical impact specimens measuring $\varphi 3 \mathrm{~mm} \times 3 \mathrm{~mm}$ through wire cutting after being processed by solid solution treatment with heating to $1040{ }^{\circ} \mathrm{C}$, holding for $40 \mathrm{~min}$, then air-cooling. In addition, it was guaranteed that the two end faces of specimens were parallel, and the surface roughness of the two end faces after polishing satisfied $R_{\mathrm{a}} \leq 1.6 \mu \mathrm{m}$.

Table 1 Sample chemical composition table (mass fraction/\%)

\begin{tabular}{llllllllll}
\hline Element & $\mathrm{C}$ & $\mathrm{Si}$ & $\mathrm{Cr}$ & $\mathrm{Ni}$ & $\mathrm{Mn}$ & $\mathrm{P}$ & $\mathrm{S}$ & $\mathrm{Mo}$ & $\mathrm{Fe}$ \\
\hline Ingredient & 0.99 & 0.70 & 18.0 & 0.40 & 0.68 & 0.03 & 0.02 & 0.59 & Bal. \\
\hline
\end{tabular}

The quasi-static compression test was conducted at room temperature $\left(T=25^{\circ} \mathrm{C}\right)$ based on the UTM5305 universal testing machine by setting the strain rates to $0.001 \mathrm{~s}^{-1}, 0.01 \mathrm{~s}^{-1}$, and $0.1 \mathrm{~s}^{-1}$, respectively. The dynamic compression test was performed based on the ALT1000 SHPB test platform by setting the temperature to $25{ }^{\circ} \mathrm{C}, 350{ }^{\circ} \mathrm{C}, 500{ }^{\circ} \mathrm{C}$, and $650{ }^{\circ} \mathrm{C}$ at average strain rates of $800 \mathrm{~s}^{-1}, 1500 \mathrm{~s}^{-1}, 2000 \mathrm{~s}^{-1}, 2500 \mathrm{~s}^{-1}, 3000 \mathrm{~s}^{-1}$, and $4000 \mathrm{~s}^{-1}$, respectively. During the test, the length of impact rods was 80 $\mathrm{mm}$ and the diameter of the pressure bar was $8 \mathrm{~mm}$; on this basis, it was adjusted to different air pressures $(0.1 \sim 0.4 \mathrm{MPa})$ to apply dynamic load to specimens at the aforementioned strain rates. The high temperature test was conducted with the aid of a synchronously assembled test system and heating was performed by using a resistance-type high-temperature furnace. To guarantee the accuracy of the test, three groups of valid test data were averaged and then analysed. During the test, the timevoltage curves at different temperatures and loading rates were recorded by using a super-dynamic strain instrument and a memory unit to record transient waves. The dynamic stress-strain curves of materials were attained by calculating the relationships between stress, strain rate, and strain of specimens according to stress wave theory.

\section{Test results and analysis}

\subsection{Stress-strain curves of 9Cr18Mo stainless steels}

Figure 1 shows the stress-strain curves in the quasi-static state (at strain rates of $0.001 \mathrm{~s}^{-1}, 0.01 \mathrm{~s}^{-1}$, and $0.1 \mathrm{~s}^{-1}$ ) at the test temperature of $25^{\circ} \mathrm{C}$. Fig. 2 shows the stress-strain curves in the dynamic state (at strain rates from $800 \mathrm{~s}^{-1}$ to $\left.4000 \mathrm{~s}^{-1}\right)$ at the test temperature of $25^{\circ} \mathrm{C}$. It can be seen from the figure that the specimens present certain strain-rate sensitivity both at both quasi-static and dynamic states, that is, the flow stresses of specimens increase by an amount that gradually decreases with increasing strain rate. The phenomenon is pronounced in the stress-strain curve in the dynamic state. The change trends of the stress-strain curves on the two conditions are relatively consistent within the plastic stage. As the strain rate is increased, the specimens enter the stage of plastic deformation early in the dynamic state. 


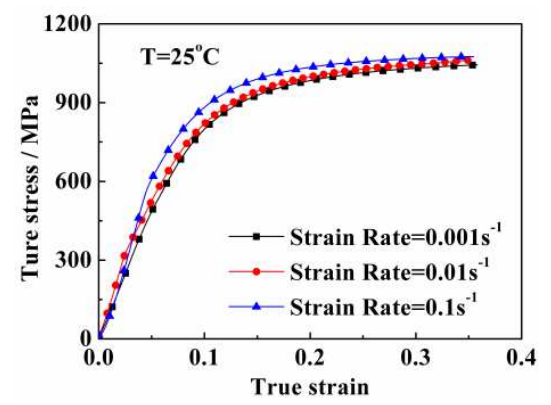

Figure 1 Stress-strain curve in quasi-static state

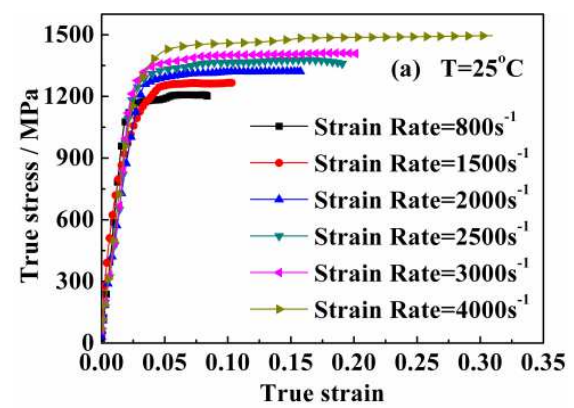

Figure 2 Stress-strain curves at different strain rates $\left(\mathrm{T}=25^{\circ} \mathrm{C}\right)$

\subsection{Strain-rate sensitivity}

As shown in Figs 1 and 2, the specimens exhibit a certain strain-rate sensitivity. To explore the influence of the strain rate on the flow stress on specimens, a sensitivity parameter $(\beta)$ of the strain rate is introduced to quantify the strain-rate strengthening effect at the given temperature, which is defined as follows ${ }^{[16-17]}$ :

$$
\beta=\frac{\partial \sigma}{\partial \ln \dot{\varepsilon}}=\frac{\sigma_{2}-\sigma_{1}}{\ln \left(\dot{\varepsilon}_{2}-\dot{\varepsilon}_{1}\right)}
$$

where, $\sigma_{1}$ and $\sigma_{2}$ refer to the corresponding flow stresses at the strain rates $\dot{\varepsilon}_{1}$ and $\dot{\varepsilon}_{2}$ at the same temperature, respectively, in which $\dot{\varepsilon}_{1}$ and $\dot{\varepsilon}_{2}$ denote two strain rates at the same temperature, respectively.

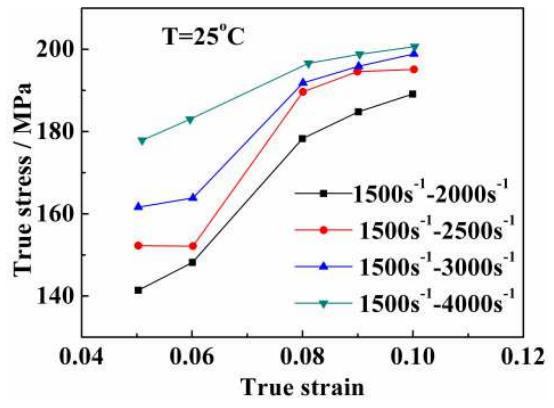

Figure 3 The relationship between strain rate sensitivity parameter $(\beta)$ and strain rate and strain

Figure 3 shows the change relationship of the sensitivity parameter $(\beta)$ of the strain rate with the strain rate and strain calculated by Equation (1) at a temperature of $25^{\circ} \mathrm{C}$. It can be seen from the figure that the sensitivity parameter $(\beta)$ of the strain rate increases asymptotically with increasing strain rate and strain: this implies that the strain-rate strengthening effect is inhibited by partial thermal softening induced by the adiabatic temperature rise during plastic deformation at a high strain rate, which leads to the reduction of the strain-rate strengthening effect. This is manifest as asymptotic growth of the sensitivity parameter $(\beta)$ of the strain rate with increasing strain rate and strain. Under high-speed loading, the heat generated during the plastic deformation of specimens cannot be readily diffused. In that case, the deformation is deemed to be an adiabatic process, therefore, an adiabatic temperature rise occurs during the plastic deformation of specimens, thus triggering the thermal softening effect. The adiabatic temperature rise occurring during the plastic deformation at a high strain rate can be defined as follows ${ }^{[18-20]}$ :

$$
\Delta T(\varepsilon)=\frac{\eta}{\rho C_{\mathrm{v}}} \int_{0}^{\varepsilon} \sigma \mathrm{d} \varepsilon(2)
$$

where, $\sigma, \varepsilon, \rho$, and $C_{\mathrm{v}}$ represent the true stress, true strain, material density, and specific heat capacity of the materials, 
respectively; $\eta$ denotes the work to heat conversion factor and it is thought that the plastic work is completely transferred to heat, so $\eta=1$. As for the specimens, $\rho=7.8 \times 10^{3} \mathrm{~kg} / \mathrm{m}^{3}$ and $C_{\mathbf{v}}=0.5 \mathrm{~kJ} /(\mathbf{k g K})$.

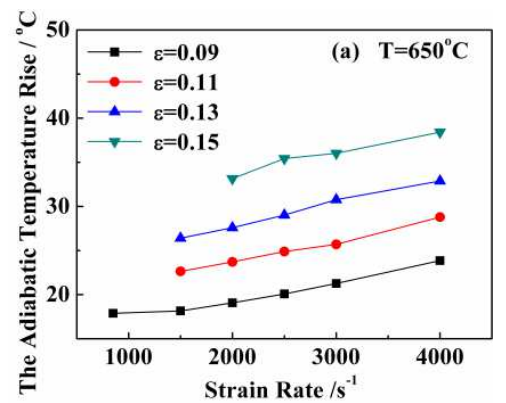

Figure 4 The relationship between adiabatic temperature rise

$$
\text { and strain } \operatorname{rate}\left(\mathrm{T}=650^{\circ} \mathrm{C}\right)
$$

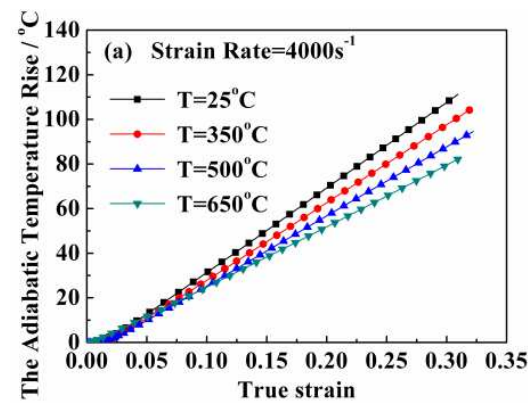

Figure 5 Adiabatic temperature rise at different temperatures $\left(\dot{\varepsilon}=4000 \mathrm{~s}^{-1}\right)$

Figure 4 shows the change in the adiabatic temperature rise at different strains with strain rate at a temperature of $650{ }^{\circ} \mathrm{C}$.

Fig. 5 shows the adiabatic temperature rises at different temperatures at a strain rate of $4000 \mathrm{~s}^{-1}$. The result shows that the adiabatic temperature rise is controlled by the strain rate and temperature: it increases with the strain rate while decreases with increasing temperature. At a given strain rate and temperature, the adiabatic temperature rise increases with the strain. This explains why the increase in sensitivity parameter $(\beta)$ of the strain rate diminishes with the increase of the strain rate and strain.

\subsection{Temperature sensitivity}

Figure 6 shows the stress-strain curves at a strain rate of $4000 \mathrm{~s}^{-1}$ when the temperature successively increases from $25{ }^{\circ} \mathrm{C}$ to $350{ }^{\circ} \mathrm{C}, 500{ }^{\circ} \mathrm{C}$ and $650{ }^{\circ} \mathrm{C}$. The result indicates that the specimens exhibit a significant thermal softening effect, that is, the flow stress gradually decreases with increasing temperature at the given strain rate; moreover, the effect is more pronounced at higher temperatures.

To quantify the influence of temperature on the flow stress at a given strain rate, the temperature sensitivity coefficient $n_{t}$ is introduced, which is defined as follows ${ }^{[21-22]}$ :

$$
n_{t}=\left|\frac{\partial \ln \sigma}{\partial \ln T}\right|=\left|\frac{\ln \left(\sigma_{2} / \sigma_{1}\right)}{\ln \left(T_{2} / T_{1}\right)}\right|(3)
$$

where, $\sigma_{1}$ and $\sigma_{2}$ refer to the stresses corresponding to a certain strain at temperatures $T_{1}$ and $T_{2}$ at the same strain rate.

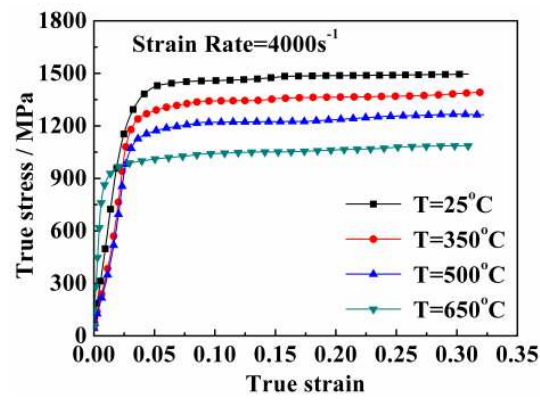

Figure 6 The stress-strain curve at different temperatures $\left(\dot{\varepsilon}=4000 \mathrm{~s}^{-1}\right)$

According to the test data obtained at a strain rate of $4000 \mathrm{~s}^{-1}$, the corresponding temperature sensitivity coefficients $\left(n_{t}\right)$ at 
strains of $0.1,0.15,0.20,0.25$, and 0.30 at temperatures $25^{\circ} \mathrm{C}, 350{ }^{\circ} \mathrm{C}, 500{ }^{\circ} \mathrm{C}$, and $650{ }^{\circ} \mathrm{C}$ are separately calculated by using Equation (3); in addition, the relationship $n_{t}$ with temperature and strain is as shown in Fig. 7. $n_{t}$ increases with the temperature by an increasing amount. As the strain increases, $n_{t}$ increases at a steady rate. This suggests that the reduction in the flow stress on specimens caused by the increasing test temperature is much greater than that induced by the adiabatic temperature rise during plastic deformation. That is, the thermal softening during plastic deformation is jointly induced by the change in test temperature and the adiabatic temperature rise during plastic deformation; nevertheless, the change in test temperature predominates in its influence of the thermal softening effect.

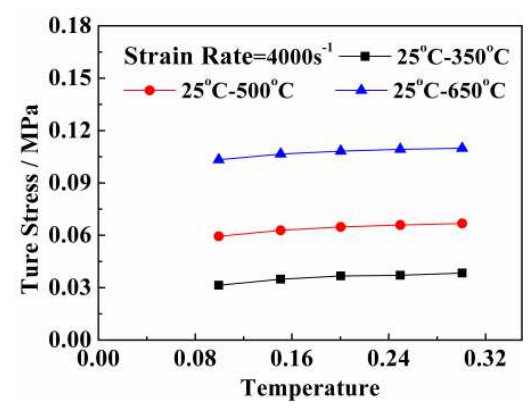

Figure 7 The relationship of temperature sensitivity with temperature and $\operatorname{strain}\left(\dot{\varepsilon}=4000 \mathrm{~s}^{-1}\right)$

\section{Establishment of the constitutive models for 9Cr18Mo stainless steel}

4.1 Establishment of the J-C constitutive model

The J-C model consists of the strain hardening terms, strain-rate strengthening term, and thermal softening term for describing the dynamic deformation process of materials, which is expressed as follows ${ }^{[6,23]}$ :

$$
\begin{gathered}
\bar{\sigma}=\left(A+B\left(\bar{\varepsilon}^{\mathrm{p}}\right)^{n}\right)\left(1+C \ln \dot{\varepsilon}^{*}\right)\left(1-\left(T^{*}\right)^{m}\right)(4) \\
T^{*}=\left(T-T_{\mathrm{m}}\right)\left(T_{\mathrm{m}}-T_{\mathrm{r}}\right)(5)
\end{gathered}
$$

where, $\bar{\sigma}, A, B, n, C$, and $m$ refer to the flow stress on materials, the yield strength at the reference temperature and reference strain rate (quasi-static state), strain hardening coefficient, strain hardening index, strain-rate strengthening coefficient, and thermal softening index, respectively; $\bar{\varepsilon}^{\mathrm{p}}=\varepsilon^{\mathrm{p}} / \varepsilon_{0}$ denotes the equivalent plastic strain, in which $\varepsilon^{\mathrm{p}}$ and $\varepsilon_{0}$ denote the plastic strain and reference strain, respectively; $\dot{\varepsilon}^{*}=\dot{\varepsilon} / \dot{\varepsilon}_{0}$ denotes the dimensionless plastic strain rate, where $\dot{\varepsilon}_{0}$ and $\dot{\mathcal{E}}$ separately refer to the reference strain rate and plastic strain rate; $T^{*}, T_{\mathrm{r}}, T_{\mathrm{m}}$ and $T$ separately represent the relative temperature, reference temperature, the melting point of the materials, and the test temperature.

(1) Determination of strain hardening terms $A, B$, and $n$

When determining the strain hardening terms, it is supposed that both the values of the strain-rate strengthening terms and thermal softening term are equal to 1 . The constitutive model is simplified as follows:

$$
\bar{\sigma}=A+B\left(\bar{\varepsilon}^{-\mathrm{p}}\right)^{n}(6)
$$

By taking the stress-strain curve in the quasi-static state $\left(\dot{\varepsilon}=0.001 \mathrm{~s}^{-1}\right)$ as the reference curve, the yield strength of 
specimens is found to be $1000.16 \mathrm{MPa}$ and the reference strain is taken as 0.01 within the transition range from the elastic zone to the plastic zone in the stress-strain curve. By rewriting Equation (6) and taking the logarithms in two sides, it can be found that:

$$
\ln (\bar{\sigma}-A)=+\ln B+n \ln \bar{\varepsilon}^{\mathrm{p}}(7)
$$

By substituting the stress and strain during the plastic deformation in the reference curve into Equation (7) and then performing linear fitting, the values of $n$ and $B$ are found to be 0.69031 and 6.614 , respectively. The result of the linear fitting is shown in Fig. 8.

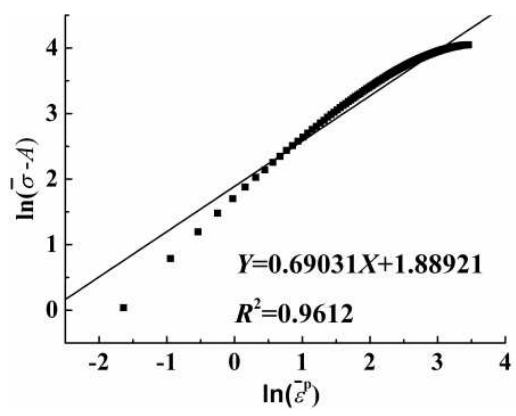

Fig. 8 The first fitting curve of the J-C mode

(2) Determination of the strain-rate strengthening coefficient $C$

It is supposed that the value of the thermal softening term at normal temperature is equal to 1 and therefore Equation (4) can be simplified as follows:

$$
\bar{\sigma}=\left(A+B\left(\bar{\varepsilon}^{\mathrm{p}}\right)^{n}\right)\left(1+C \ln \dot{\varepsilon}^{*}\right)(8)
$$

By rewriting Equation (8), it can be found that:

$$
\frac{\bar{\sigma}}{A+B\left(\bar{\varepsilon}^{\mathrm{p}}\right)^{n}}=1+C \ln \dot{\varepsilon}^{*}(9)
$$

By taking the reference strain rate as $250 \mathrm{~s}^{-1}$, the test data under the fixed strain at different strain rates are averaged and then substituted into Equation (9) to perform linear fitting. $C$ is determined as 0.14911 . The result of the linear fitting is displayed in Fig. 9.

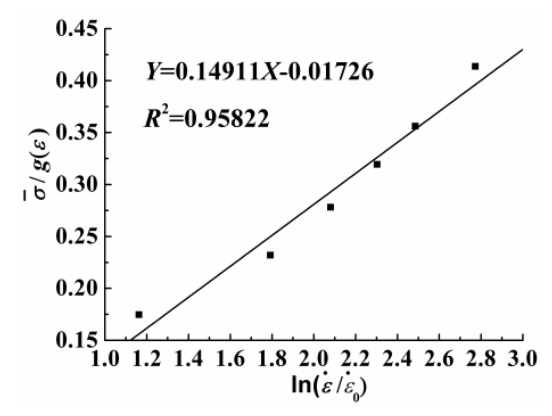

Fig. 9 The second fitting curve of the J-C model

(3) Determination of the thermal softening index $m$

By rewriting Equation (4), it can be found that: 


$$
\frac{\bar{\sigma}}{\left(A+B\left(\bar{\varepsilon}^{\mathrm{p}}\right)^{n}\right)\left(1+C \ln \dot{\varepsilon}^{*}\right)}=1-\left(T^{*}\right)^{m}(10)
$$

By rewriting Equation (10) and taking the logarithm of both sides of the equation, it is found that:

$$
\ln \left(1-\frac{\bar{\sigma}}{\left(A+B\left(\bar{\varepsilon}^{\mathrm{p}}\right)^{n}\right)\left(1+C \ln \dot{\varepsilon}^{*}\right)}\right)=m \ln T^{*}(11)
$$

By using the stress-strain curves at a strain rate of $4000 \mathrm{~s}^{-1}$ at different temperatures, the thermal softening term $m$ of materials can be determined. By setting the melting point of specimens as $T_{m}=1420^{\circ} \mathrm{C}$, the test data at a fixed strain are averaged, then substituted into Equation (11) to allow linear fitting. Furthermore, the value of $m$ is found to be 1.65204. The result of the linear fitting is shown in Fig. 10.

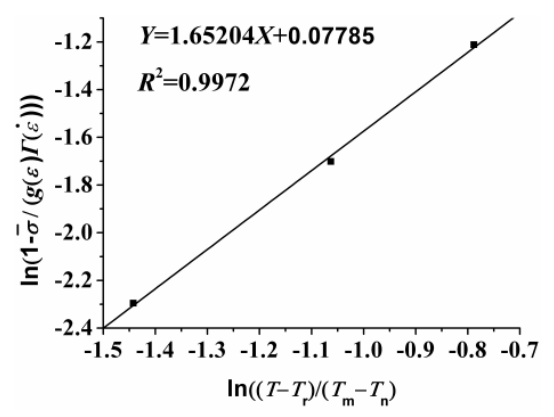

Fig. 10 The third fitting curve of the J-C model

Thus, the J-C constitutive model for describing 9Cr18Mo stainless steel is expressed as follows:

$$
\bar{\sigma}=\left(1000.16+6.614\left(\frac{\varepsilon^{\mathrm{p}}}{0.01}\right)^{0.69031}\right)\left(1+0.14911 \ln \left(\frac{\dot{\varepsilon}}{250}\right)\right)\left(1-\left(\frac{T-25}{1375}\right)^{1.65204}\right)^{(12)}
$$

4.2 Establishment of the P-L constitutive model

The P-L constitutive model is commonly used to describe the change relationships of the flow stress on materials at high temperature, high strain rate, and large strain with temperature, strain rate, and strain, as given by ${ }^{[24-25]}$ :

$$
\begin{gathered}
\sigma\left(\varepsilon_{s}, \dot{\varepsilon}_{s}, T\right)=g\left(\varepsilon_{s}\right) \Gamma\left(\dot{\varepsilon}_{s}\right) \Theta(T)(13) \\
g\left(\varepsilon_{s}\right)=\sigma_{0}\left(1+\frac{\varepsilon_{s}}{\sigma_{0}}\right)^{1 / n}(14) \\
\Gamma\left(\dot{\varepsilon}_{s}\right)=\left(1+\frac{\dot{\varepsilon}_{s}}{\dot{\varepsilon}_{0}}\right)^{1 / m}(15) \\
\Theta(T)=C_{0}+C_{1} T+C_{2} T^{2}+C_{3} T^{3}+C_{4} T^{4}+C_{5} T^{5}(16) \\
T=T_{\text {in }}+\Delta T(17)
\end{gathered}
$$

where, $n, m, T_{\text {in }}, \Delta T$, and $\sigma_{0}$ denote the strain hardening index, strain-rate strengthening index, initial test temperature, adiabatic temperature rise, and the yield strength at the reference temperature and reference strain rate, respectively; 
$\varepsilon_{s}, \varepsilon_{0}, \dot{\varepsilon}_{S}$, and $\dot{\varepsilon}_{0}$ refer to the plastic strain, reference strain, plastic strain rate, and reference strain rate, respectively;

$C_{0}, C_{1}, \ldots$, and $C_{5}$ separately represent the polynomial coefficients of temperature.

(1) Determination of strain hardening index $n$

When constructing the constitutive model, it is supposed that the strain term $\left(g\left(\varepsilon_{s}\right)\right)$, strain rate term $\left(\Gamma\left(\dot{\varepsilon}_{s}\right)\right)$, and temperature term $(\Theta(T))$ are mutually independent. When determining the corresponding parameters in the strain term $g\left(\varepsilon_{s}\right)$, the strain rate term $\Gamma\left(\dot{\varepsilon}_{s}\right)$ and temperature term $\Theta(T)$ are set to 1, thus, Equation (13) can be simplified to:

$$
\sigma\left(\varepsilon_{s}\right)=g\left(\varepsilon_{s}\right)=\sigma_{0}\left(1+\frac{\varepsilon_{s}}{\sigma_{0}}\right)^{1 / n(18)}
$$

The stress-strain curve in the quasi-static state $\left(\dot{\varepsilon}=0.001 \mathrm{~s}^{-1}\right)$ is taken as the reference curve and the reference strain is set as 0.001. On this basis, by rewriting Equation (18) and taking the logarithms of each side of the equation, it can be found that:

$$
\ln \left(\frac{\sigma\left(\varepsilon_{s}\right)}{\sigma_{0}}\right)=\frac{1}{n} \ln \left(1+\frac{\varepsilon_{s}}{\varepsilon_{0}}\right)(19)
$$

By substituting the corresponding stress after the strain of 0.39 and the corresponding strain in the reference curve into the above equation and then performing the linear fitting, the slope $1 / n$ of the curve is calculated as 0.02459 ; furthermore, the strain hardening index $n$ is found to be 40.66693778. The result of the linear fitting is displayed in Fig. 11.

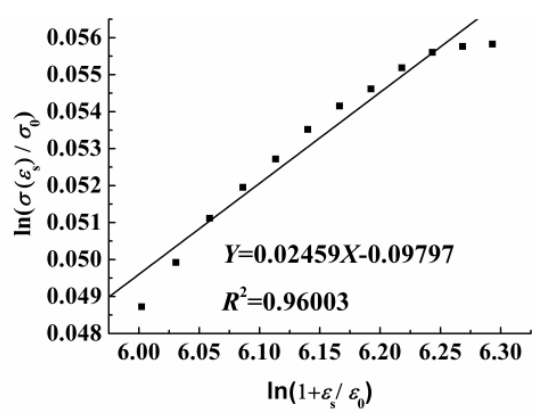

Fig. 11 Relationship between $\ln \left(\sigma\left(\varepsilon_{s}\right) / \sigma_{0}\right)$ and $\ln \left(1+\varepsilon_{s} / \varepsilon_{0}\right)$

(2) Determination of the strain-rate strengthening index $m$

The strain-rate strengthening index $m$ is determined by utilising the stress-strain curve at strain rates of $800 \sim 4000 \mathrm{~s}^{-1}$ at $25^{\circ} \mathrm{C}$. It is supposed that the thermal softening term $\Theta(T)$ is equal to 1 at that temperature and therefore Equation (13) can be simplified to:

$$
\sigma\left(\varepsilon_{s}, \dot{\varepsilon}_{s}\right)=g\left(\varepsilon_{s}\right)\left(1+\frac{\dot{\varepsilon}_{s}}{\dot{\varepsilon}_{0}}\right)^{1 / m}(20)
$$

By taking the logarithm of each side of the above equation, it is found that:

$$
\ln \left(\frac{\sigma\left(\varepsilon_{s}, \dot{\varepsilon}_{s}\right)}{g\left(\varepsilon_{s}\right)}\right)=\frac{1}{m} \ln \left(1+\frac{\dot{\varepsilon}_{s}}{\dot{\varepsilon}_{0}}\right)(21)
$$

By setting the reference strain rate to $2300 \mathrm{~s}^{-1}$, the test data at a constant strain are averaged, then substituted into Equation 
(21). Linear fitting is conducted based on the least squares method to determine the slope $1 / m$ as 0.25602 . Furthermore, the strain-rate strengthening index $m$ is calculated as 3.906. The fitting result is shown in Fig. 12.

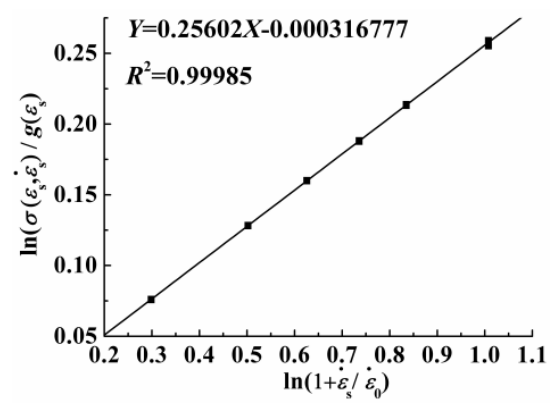

Fig. 12 Relationship between $\ln \left(\sigma\left(\varepsilon_{s}, \dot{\varepsilon}_{s}\right) / g\left(\varepsilon_{s}\right)\right)$ and $\ln \left(1+\dot{\varepsilon}_{s} / \dot{\varepsilon}_{0}\right)$

(3) Determination of the thermal softening coefficients $C_{0}, C_{1}, \ldots, C_{5}$

The thermal softening coefficients $C_{0}, C_{1}, \ldots$, and $C_{5}$ are determined based on the stress-strain curves at strain rates from $800 \sim 4000 \mathrm{~s}^{-1}$ and at temperatures from $25 \sim 650{ }^{\circ} \mathrm{C}$. Equation (13) is rewritten as follows:

$$
\frac{\sigma\left(\varepsilon_{s}, \dot{\varepsilon}_{s}, T\right)}{g\left(\varepsilon_{s}\right) \Gamma\left(\dot{\varepsilon}_{s}\right)}=C_{0}+C_{1} T+C_{2} T^{2}+C_{3} T^{3}+C_{4} T^{4}+C_{5} T^{5(22)}
$$

According to the above analysis, it can be seen that the adiabatic temperature rise $\Delta T$ during plastic deformation in the dynamic state shows a certain influence on the stress-strain curve, therefore, when fitting the thermal softening coefficients, it is necessary to consider the adiabatic temperature rise in the plastic deformation stage. The test data under a fixed strain at different temperatures are averaged, then substituted into Equation (22); moreover, polynomial fitting is performed to attain the values of $C_{0}(1.00824), C_{1}\left(2.38267 \times 10^{-5}\right)$ and $C_{2}\left(-6.91782 \times 10^{-7}\right)$. The values of $C_{3}, C_{4}$ and $C_{5}$ approximate to 0 . The fitting curve is shown in Fig. 13.

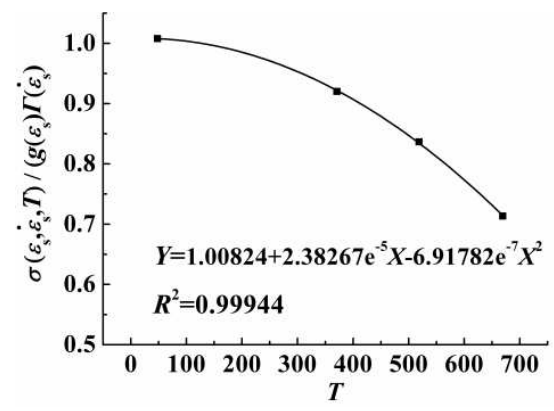

Fig. 13 Relationship between $\sigma\left(\varepsilon_{s}, \dot{\varepsilon}_{s}, T\right) / g\left(\varepsilon_{s}\right) \Gamma\left(\dot{\varepsilon}_{s}\right)$ and $T$

Hence, the P-L constitutive model for describing 9Cr18Mo stainless steel is expressed as follows:

$$
\begin{aligned}
& \sigma\left(\varepsilon_{s}, \dot{\varepsilon}_{s}, T\right)=g\left(\varepsilon_{s}\right) \Gamma\left(\dot{\varepsilon}_{s}\right) \Theta(T) \\
& =1000.16\left(1+\frac{\varepsilon_{s}}{0.001}\right)^{0.02459}\left(1+\frac{\dot{\varepsilon}_{s}}{2300}\right)^{0.25602} \\
& \left(1.00824+2.38267 \times 10^{-5}-6.91782 \times 10^{-7}\right)
\end{aligned}
$$

\section{Prediction and comparison of constitutive models}

Figure 14 compares the predicted values obtained through two constitutive models with test values under different test conditions. Table 2 lists the mean values of absolute errors incurred when using the two constitutive models. As shown in Fig. 
14 and Table 2, the predicted values obtained through two constitutive models both present a certain prediction error with increasing strain rate and temperature. To be specific, the error rises at first and then declines with increasing strain rate and temperature. The mean value of the absolute error predicted by the $\mathrm{J}$-C constitutive model reaches a maximum (51.544) at a strain rate of $2000 \mathrm{~s}^{-1}$ and temperature of $650{ }^{\circ} \mathrm{C}$; the maximum mean value (46.367) of the absolute error predicted by the P-L constitutive model is found at a strain rate of $2500 \mathrm{~s}^{-1}$ and a temperature of $500{ }^{\circ} \mathrm{C}$. It can be seen from Table 2 that the prediction accuracy of the P-L constitutive model is higher than that of the $\mathrm{J}-\mathrm{C}$ constitutive model. This is because the thermal softening effect caused by adiabatic temperature rise is considered in the P-L constitutive model when determining polynomial coefficients of temperature.
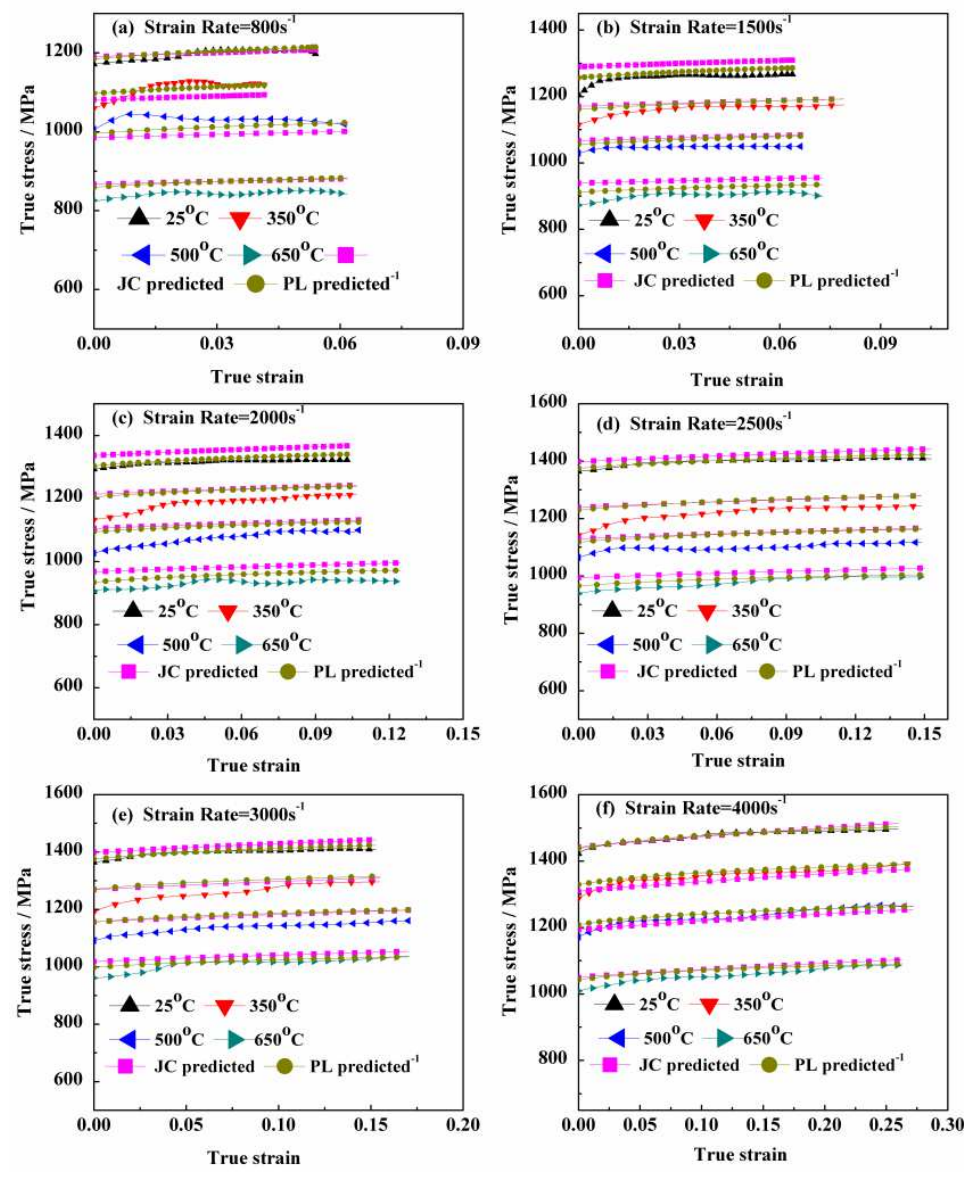

Fig. 14: Comparison of experimental values of stress-strain curves with model predictions under different strain rates 
Table 2 Average absolute error of two constitutive models under different experimental conditions

\begin{tabular}{|c|c|c|c|}
\hline$T /{ }^{\circ} \mathrm{C}$ & $\dot{\varepsilon} / \mathrm{s}^{-1}$ & $\Delta \sigma_{\mathrm{JC}} / \mathrm{MPa}$ & $\Delta \sigma_{\mathrm{PL}} / \mathrm{MPa}$ \\
\hline \multirow{6}{*}{25} & 800 & 6.424471638 & 6.109457399 \\
\hline & 1500 & 38.98703638 & 15.33518007 \\
\hline & 2000 & 37.04814443 & 9.391618684 \\
\hline & 2500 & 29.46215782 & 5.722001128 \\
\hline & 3000 & 22.34984852 & 5.923465121 \\
\hline & 4000 & 5.982440216 & 3.902910575 \\
\hline \multirow{6}{*}{350} & 800 & 27.04190337 & 10.60611432 \\
\hline & 1500 & 20.1336439 & 18.2893326 \\
\hline & 2000 & 40.92181462 & 37.65429567 \\
\hline & 2500 & 42.34938154 & 41.79854765 \\
\hline & 3000 & 29.80158046 & 36.78908546 \\
\hline & 4000 & 12.46319636 & 11.87826906 \\
\hline \multirow{6}{*}{500} & 800 & 32.79624831 & 17.3101421 \\
\hline & 1500 & 28.39327725 & 23.19058462 \\
\hline & 2000 & 45.03536918 & 39.11484364 \\
\hline & 2500 & 49.39455932 & 46.36667909 \\
\hline & 3000 & 41.94325487 & 45.30685472 \\
\hline & 4000 & 9.845891459 & 10.62832331 \\
\hline \multirow{6}{*}{650} & 800 & 31.25790377 & 30.58632256 \\
\hline & 1500 & 49.19664884 & 27.14529 \\
\hline & 2000 & 51.54404536 & 26.74278264 \\
\hline & 2500 & 33.93195871 & 11.88201571 \\
\hline & 3000 & 26.76224827 & 9.985216708 \\
\hline & 4000 & 20.68909126 & 15.5467803 \\
\hline
\end{tabular}

The further to explore the prediction accuracy of the two constitutive models, two parameters (AARE and $R$ ) are introduced ${ }^{[26-29]}$ :

$$
\begin{gathered}
A A R E=\frac{1}{N} \sum_{i=1}^{N}\left|\frac{E_{i}-P_{i}}{E_{i}}\right| \\
R=\frac{\sum_{i=1}^{N}\left(E_{i}-\bar{E}\right)\left(P_{i}-\bar{P}\right)}{\sqrt{\sum_{i=1}^{N}\left(E_{i}-\bar{E}\right)^{2} \sum_{i=1}^{N}\left(P_{i}-\bar{P}\right)^{2}}}
\end{gathered}
$$

where, $E_{i}$ and $P_{i}$ separately refer to test and predicted values of the flow stresses (MPa); $\bar{E}$ and $\bar{P}$ separately represent mean values of $E_{i}$ and $P_{i} ; N$ denotes for the total number of data points in the study.

The $R$ values and AAREs of the two constitutive models are separately calculated according to the test data and the predicted values by the models; the results are shown in Fig. 15 and Table 3. The $R$ values are 0.9697 and 0.9896 while the AAREs of the values predicted by the J-C and P-L constitutive models are $2.77 \%$ and $1.85 \%$ when compared with the test values, respectively. 
Therefore, it can be found that the P-L constitutive model delivers a higher prediction accuracy, which can more accurately describe the flow stress in $9 \mathrm{Cr} 18 \mathrm{Mo}$ stainless steel and the changes thereto with strain, strain rate, and temperature.
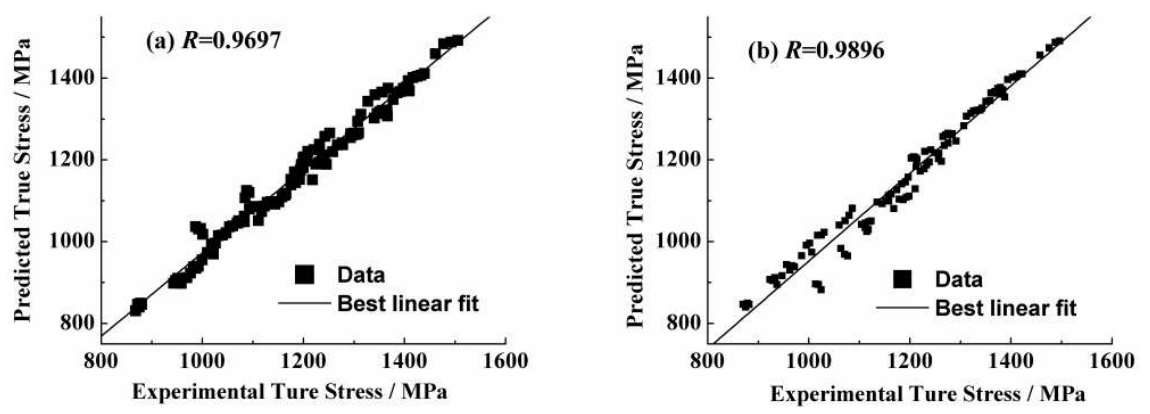

Fig. 15: The correlation between experimental and predicted values of two constitutive models

Table 3 The average relative error of the two constitutive models

\begin{tabular}{ccl}
\hline Model & Johnson-Cook & Power-Law \\
\hline AARE & 0.0277 & 0.0185 \\
\hline
\end{tabular}

\section{Conclusion}

(1) The 9Cr18Mo stainless steel shows a certain strain-rate strengthening effect and significant thermal softening: the flow stress on $9 \mathrm{Cr} 18 \mathrm{Mo}$ stainless steel gradually increases with increasing strain rate while it decreases significantly with increasing temperature.

(2) The $R$ values are 0.9697 and 0.9896 while AAREs of the two constitutive models are $2.77 \%$ and $1.85 \%$, respectively: the P-L constitutive model exhibits a higher prediction accuracy than the J-C constitutive model and it can better describe the rheological behaviours of 9Cr18Mo stainless steel at high temperatures and high strain rates.

(3) The prediction accuracies of both constitutive models present a certain error with increasing temperature and strain rate, which is related to the high non-linearity of the flow stress on specimens at high temperatures and high strain rates.

\section{References}

[1] Z.B. Liu, J.X. Liang, J. S, X.H. Wang, Y.Q. Sun, C.J. Wang, Z.R. Yang. Research and Application Progress in UltraHighStrength Stainless Steel. Acta Metallurgica Sinica.56,549-557(2020).

[2] Ben Young, Wing-Man Lui. Behavior of Cold-Formed High Strength Stainless Steel Sections. J. Struct. Eng.131,17381745(2005).

[3] Ehab Ellobodya, Ben Young, Structural performance of cold-formed high strength stainless steel columns.Journal of Constructional Steel Research. 61,1631-1649(2005).

[4] Z.Y. Yang, Z.B. Liu, J.X. Liang, Y.Q. Sun, W.H. Li. Development of maraging stainless steel. Journal of Materials and Heat Treatment. 29,1-7( 2008).

[5] F. J. Zerilli, R. W. Armstrong. Dislocation-mechanics-based Constitutive Relations for Material Dynamics Calculations. J. Appl. Phys.61,1816-1825(1987).

[6] G. R. Johnson, W. H. Cook.A constitutive model and data for metals subjected to large strains ,and high temperatures ,Engineering Fracture Mechanics.21,541-548(1983).

[7] Dipti Samantaray, Sumantra Mandal, Utpal Borah, A.K. Bhaduri, P.V. Sivaprasad. A thermo-viscoplastic constitutive model to predict clevated-temperature flow behaviour in a titanium-modified austenitic stainless steel. Materials Science and Engineering A. 526,1-6(2009). 
[8] J.X. Kong, H. Chen, N. He, L. LI, F.Jiang. Dynamic mechanical properties test and constitutive model of pure iron material . Acta Aeronautica et Astronautica Sinica. 35,2063-2071(2014).

[9] S.Tanimura,T.Tsuda, A.Abe,H.Hayashi, N.Jones. Comparison of rate-dependent constitutive models with experimental data. International Journal Impact Engineering. 69,104-113(2014).

[10] Z. He, S.G. Zhao, J.L. Yang, W. Cheng. Experimental Investigation of the Dynamic Material Property of Stainless Steel:0Cr17Ni4Cu4Nb.Journal of Materials Science and Engineering. 25,418-421(2007).

[11] H.Z. Yan, L.J. Gong. Constitutive model and finite element simulation of 20CrMo material. Journal of Central South University (Science and Technology).43, 4268-4273 (2012).

[12] D. Forni, B. Chiaia, E. Cadoni. Strain rate behaviour in tension of S355 steel: Base for progressive collapse analysis. Engineering Structures. 119,164-173(2016).

[13] B. J. Tuazon, K.-O. Bae, S.-H. Lee, H.-S. Shin. Integration of a new data acquisition/processing scheme in SHPB test and characterization of the dynamic material properties of high-strength steels using the optional form of Johnson-Cook model. Journal of Mechanical Science and Technology. 28,3561-3568 (2014).

[14] Yong Huang, Steven Y. Liang. Modelling of CBN tool crater wear in finish hard turning. The International Journal of Advanced Manufacturing Technology. 24,632-639(2004).

[15] D. Samantaray, S. Mandal, A. K. Bhaduri, P. V. Sivaprasad. An overview on constitutive modelling to predict elevated temperature flow behaviour of fast reactor structural materials . Transactions of the Indian Institute of Metals. 63,823-831(2010).

[16] H.J. Zhou, M.Z. Huang. Strength of Metallic Materials.168-169( Science Press,Beijing, 1989)

[17] Y. Nan, Y.Q. Ning, H.Q. Liang, H.Z. Guo, Z.K. Yao, M.W. Fu. Work-hardening effect and strain-rate sensitivity behavior during hot deformation of Ti-5Al-5Mo-5V-1Cr-1Fe alloy. Materials \& Design. 82,84-90(2015).

[18] Y.F. Lie, X.G. Zeng. Determination of Conversion Factor in Adiabatic Temperature Rise of Dynamic Tensile Behavior of TC11 Alloy . Rare Metal Materials and Engineering.47,2056-2060(2018).

[19] Q.F. Dai, R.B. Song, W.Y. Fan, Z.F. Guo, X.X. Guan. Behaviour and mechanism of strain hardening for dual phase steel DP1180 under high strain rate deformation. Acta Metallurica Sinica.48,1160-1165(2012).

[20] L.L. Wang, Progress in impact dynamics. (University of Science and Technology of China Press,Hefei,1992)

[21] Z.Xu,Y.Li. Dynamic behaviors of 0Cr18Ni10Ti stainless steel welded joints at elevated temperatures and high strain rates. Mechanics of Materials. 41, 121-130(2009).

[22] Woei-Shyan Lee,Tao-Hsing Chen,Chi-Feng Lin,Wen-Zhen Luo. Dynamic Mechanical Response of Biomedical 316L Stainless Steel as Function of Strain Rate and Temperature. Bioinorganic Chemistry and Applications. 2011, 113(2011).

[23] C. Shu, L. Cheng, Y. Xu. Research on parameter estimation of Johnson-Cook constitutive model, The Chinese Journal of Nonferrous Metals.30, 1073-1083(2020).

[24] Y.H. Zhao, J. Sun, J.F. Li, Y.Q. Yan, P. Wang. A comparative study on Johnson-Cook and modified Johnson-Cook constitutive material model to predict the dynamic behavior laser additive manufacturing FeCr alloy, J. Alloys Compd.723,179-187(2017).

[25] J.C. Yu,F. Jiang, Y.M. Rong,H. Xie,T. Suo, Numerical study the flow stress in the machining process. The International Journal of Advanced Manufacturing Technology. 74,509-517(2014).

[26] B. Zhang,L. Yue,H.F. Chen,Z.J. Zhang,P.R. Liu,F.F. Zhao. Hot deformation behavior of as-cast GH4169 alloy and comparison of three constitutive models.Rare Metal Materials and Engineering. 50,212-222(2021).

[27] Y. Liu, M. Li, X.W. Ren, Z.B. Xiao, X.Y. Zhang, Y.C. Huang. Flow stress prediction of Hastelloy C-276 alloy using modified Zerilli-Armstrong,Johnson-Cook and Arrhenius-type constitutive models. Trans.Nonferrous Met.Soc.China. 30,3031-3042(2020).

[28] D.G. Niu, C. Zhao, D.X. Li, Z. Wang, Z.Q. Luo, W.W. Zhang, Constitutive Modeling of the Flow Stress Behavior for the Hot Deformation of Cu-15Ni-8Sn Alloys. Frontiers in Materials 7, (2020).

[29] Amir Hossein Sheikhali,Maryam Morakkabati,Seyed Mahdi Abbasi. Constitutive Modeling for Hot Working

Behavior of SP-700 Titanium Alloy. Journal of Materials Engineering and Performance. 28, 6525-6537(2019). 